\title{
Evaluación de Cuatro Protocolos de Anestesia sobre las Variaciones en las Funciones Vitales en la Ovariohisterectomía Canina
}

\author{
Evaluation of Four Protocols of Anesthesia on Changes in Vital \\ Functions in the Canine Ovariohysterectomy
}

Jhon David Huayta Huanca ${ }^{1,2}$

\section{Resumen}

El presente estudio tuvo como objetivo la evaluación de los efectos de cuatro protocolos de anestesia sobre las funciones vitales de frecuencia cardiaca, pulso, frecuencia respiratoria, saturación de oxígeno y temperatura corporal en la ovariohisterectomía canina. Se seleccionaron 32 perras adultas (1-8 años), clínicamente sanas, sin distinción de peso ni raza, y clasificadas como ASA I $(84.4 \%)$ o II (15.6\%) para ser sometidas a cirugía electiva (no emergencias). Los canes fueron distribuidos en cuatro grupos de ocho individuos, donde cada grupo fue sometido a un protocolo anestésico específico. Protocolo 1: Premedicación con acepromacina y morfina; inducción con diazepam y ketamina; mantenimiento con xilacina; Protocolo 2: Inducción con diazepam y ketamina; mantenimiento con xilacina y ketamina; Protocolo 3: Inducción con propofol y diazepam; mantenimiento con propofol y fentanilo; Protocolo 4: Inducción con propofol; mantenimiento con isoflurano y fentanilo. La recolección de datos se hizo durante cuatro periodos del procedimiento quirúrgico: preoperatorio, inducción, mantenimiento y término de la cirugía. Se encontró diferencia estadística $(\mathrm{p}<0.05)$ para frecuencia cardiaca durante el periodo de inducción entre el protocolo 4 con los protocolos 1 y 2 y para el periodo de mantenimiento entre los protocolos 3 y 4 ; en pulso durante el periodo de mantenimiento entre los protocolos 3 y 4; en saturación de oxígeno en el periodo de mantenimiento y término de la cirugía entre protocolos, excepto entre protocolos 1 y 2 ; y en temperatura corporal en los periodos de inducción y mantenimiento entre los protocolos 1 y 2. Clínicamente, los registros de frecuencia cardiaca, pulso y frecuencia respiratoria mostraron mayores cambios desfavorables en el protocolo 2 (taquicardias, bradicardias, pulsos hipocinéticos y taquipneas). Asimismo, se presentó hipoxemia muy grave en el protocolo

\footnotetext{
${ }^{1}$ Escuela Profesional de Medicina Veterinaria y Zootecnia, Facultad de Ciencias e Ingenierías Biológicas y Químicas, Universidad Católica de Santa María, Arequipa, Perú

${ }^{2}$ E-mail: david_16_90@hotmail.com
} 
3, e hipotermia en todos los grupos, especialmente en el protocolo 1. Se obtuvieron planos anestésicos quirúrgicos adecuados para los protocolos 1 y 3 . Se concluye que el protocolo 1 es el más adecuado en procedimientos quirúrgicos de ovariohisterectomía canina.

Palabras clave: protocolos, anestesia, ovariohisterectomía, caninos, inducción, hipoxemia

\section{Abstract}

The aim of this study was to evaluate the effects of four protocols of anesthesia on vital functions (heart rate, pulse, respiratory rate, body temperature, and oxygen saturation) in the canine ovariohysterectomy. Thirty two bitches (1-8 years), clinically healthy, without distinction of breed and body weight, and classified as ASA I (84.4\%) and II (15.6\%) were submitted to elective surgery (not emergencies). The animals were distributed in four groups where each group was object of a specific anesthetic protocol. Protocol 1: Premedication with acepromazine and morphine; induction with diazepam and ketamine; maintenance with xylazine; Protocol 2: induction with diazepam and ketamine; maintenance with xylazine and ketamine; Protocol 3: Induction with propofol and diazepam; maintenance with propofol and fentanyl; Protocol 4: Induction with propofol; maintenance with isoflurane and fentanyl. Data collection was done during four periods of the surgical procedure: pre-surgery, induction, maintenance, and end of the surgery. There was statistical difference $(\mathrm{p}<0.05)$ on heart rate during the induction period between protocol 4 and protocols 1 and 2 and in the maintenance period between protocols 3 and 4; on pulse between protocols 3 and 4 during the maintenance period; on oxygen saturation during maintenance period and end of the surgery between protocols, except between protocols 1 and 2; and on temperature between protocols 1 and 2 during the induction and maintenance periods. Clinically, records of heart rate, pulse, and respiratory rate showed major unfavorable changes in protocol 2 (tachycardia, bradycardia, hypokinetic pulses and tachypnea). Highly severe hypoxemia was observed in protocol 3 and hypothermia in all groups but especially in protocol 1. Adequate surgical anesthetic plane was obtained in protocols 1 and 3. It is concluded that protocol 1 is the most suitable for surgical procedures of canine ovariohysterectomy.

Key words: protocols, anesthesia, ovariohysterectomy, canine, induction, hypoxemia

\section{INTRODUCCIÓN}

La ovariohisterectomía es uno de los procedimientos quirúrgicos de mayor demanda en la clínica de animales de compañía y por ello se ha convertido en uno de los procesos de rutina en la práctica de la Medicina Veterinaria. Este procedimiento, no obstante, conlleva riesgos como todo proceso quirúrgico, siendo uno de ellos el procedimiento anestésico (Burzaco y Martínez, 2001; Rioja et al., 2013) al que es sometido el paciente.
La anestesia general consiste en un estado de inconciencia causado por fármacos y que se caracteriza por ser controlable, reversible y destinado a que el animal no responda a estímulos dolorosos. Las funciones vitales deben estar disminuidas, mas no ausentes (Rioja et al., 2013).

Uno de los pilares para que el proceso quirúrgico se lleve con éxito es la eficacia de la anestesia quirúrgica (Thurmon et al., 2003). En la actualidad no existen fármacos anestésicos que produzcan los tres compo- 
nentes necesarios (inconciencia, relajación muscular y analgesia) para una adecuada anestesia quirúrgica, pero ese estado se puede obtener mediante la combinación de fármacos, existiendo una amplia gama de combinaciones entre estos compuestos.

La anestesiología es una rama importante de la Medicina Veterinaria; sin embargo, no se cuentan con estudios en el país que respalden la eficacia de una combinación determinada de fármacos anestésicos en pacientes caninos. Trabajos experimentales en esta área han sido realizados en Argentina (Otero et al., 2000), Brasil (Da Silva et al., 2000), Chile (Thibaut et al., 2002) y Colombia (Báez et al., 2007; Peña et al., 2007). Por ello, el objetivo del presente estudio fue la evaluación de cuatro protocolos de anestesia, utilizando fármacos disponibles en el país, sobre las variaciones de las funciones vitales en la ovariohisterectomía canina.

\section{Materiales y Métodos}

\section{Animales}

El estudio se llevó a cabo entre enero y abril de 2015 en la Clínica Veterinaria Terán, localizada en la ciudad de Arequipa, Perú.

Se seleccionaron perras de 1 a 8 años, sin peso ni raza específica y cuyos dueños las llevaron a la clínica para cirugía de esterilización programada o electiva (no emergencias). Asimismo, se consideró que los animales no debían estar en la fase de celo. Se seleccionaron 32 hembras clínicamente sanas, clasificadas con factor de riesgo anestésico como ASA I y ASA II (ASA, 2014). El estudio fue respaldado por el Comité de Ética de la Investigación de la Universidad Católica de Santa María.

Se formaron cuatro grupos, cada uno de ellos constituido de ocho pacientes, para la evaluación de cuatro protocolos de anestesia.

\section{Protocolos Anestésicos}

Protocolo 1

- Premedicación: Acepromacina maleato (Tranquiliss, Biomont) a dosis de $0.05 \mathrm{mg}$ / kg IM y clorhidrato de morfina (Digemid) a dosis de $0.5 \mathrm{mg} / \mathrm{kg} \mathrm{IM}$.

- Inducción: Diazepam (Diazepet, Brouwer) a dosis de $0.5 \mathrm{mg} / \mathrm{kg}$ IV y clorhidrato de ketamina (Ket-A-100, Agrovet Market) a dosis de $10 \mathrm{mg} / \mathrm{kg}$ IV.

- Mantenimiento: Clorhidrato de xilacina (Dormi-Xyl, Agrovet Market) a dosis de $0.5 \mathrm{mg} / \mathrm{kg} / \mathrm{h}$ como infusión continua.

\section{Protocolo 2}

- Inducción: Diazepam (Diazepet, Brouwer) a dosis de $0.5 \mathrm{mg} / \mathrm{kg}$ IV y clorhidrato de ketamina (Ket-A-100, Agrovet Market) a dosis de $10 \mathrm{mg} / \mathrm{kg}$ IV.

- Mantenimiento: Clorhidrato de xilacina (Dormi-Xyl, Agrovet Market) a dosis de $0.5 \mathrm{mg} / \mathrm{kg} / \mathrm{h}$ como infusión continua $\mathrm{y}$ clorhidrato de ketamina (Ket-A-100, Agrovet Market) a dosis de $2 \mathrm{mg} / \mathrm{kg} / \mathrm{h}$ como infusión continua.

\section{Protocolo 3}

- Inducción: Propofol (Fresenius Kabi) a dosis de 2-6 mg/kg vía IV y diazepam (Diazepet, Brouwer) a dosis de $0.5 \mathrm{mg} / \mathrm{kg}$ IV.

- Mantenimiento: Propofol (Fresenius Kabi) a dosis de $0.3 \mathrm{mg} / \mathrm{kg} / \mathrm{min}$ como infusión continua y fentanilo (Lab. Sanderson) a dosis de $15 \mu \mathrm{g} / \mathrm{kg} / \mathrm{h}$ como infusión continua. Se usó como bolo de ataque $5 \mu \mathrm{g} / \mathrm{kg}$ de fentanilo vía IV.

\section{Protocolo 4}

- Inducción: Propofol (Fresenius Kabi) a dosis de 2-6 mg/ $\mathrm{kg}$ vía IV.

- Mantenimiento: Isoflurano (Isoflurano, Piramal Healthcare) a concentración de $1-1.25 \%$ y fentanilo (Fentanilo, Lab. Sanderson) a dosis de $15 \mu \mathrm{g} / \mathrm{kg} / \mathrm{h}$ como infusión continua. Se usó como bolo de ataque $5 \mu \mathrm{g} / \mathrm{kg}$ de fentanilo vía IV. 
La dosis de inducción realizadas con propofol fueron condicionadas a dosis efecto, hasta alcanzar el plano anestésico quirúrgico deseado.

\section{Funciones Fisiológicas}

La evaluación clínica se llevó a cabo mediante la monitorización de las funciones fisiológicas de frecuencia cardiaca (FC), saturación de oxígeno $\left(\mathrm{SpO}_{2}\right)$ y pulso, utilizándose electrodos de tres canales en el paciente y pulsioxímetro en la mucosa de la lengua, visualizándose en un monitor multiparámetros HP (Hewlett Packard M1275A). Además, se evaluó la frecuencia respiratoria (FR) de forma visual y por auscultación, y la temperatura $\left(\mathrm{T}^{\circ}\right)$ con el uso de un termómetro digital.

Las mediciones se realizaron durante la ovariohisterectomía, y para lo cual se dividió en cuatro periodos:

- La primera medición se realizó en el periodo preoperatorio, donde no se aplicaron fármacos. Posterior a la medición de las funciones fisiológicas, se aplicó ampicilina (20 mg/kg, IV), amikacina $(12.5 \mathrm{mg} / \mathrm{kg}, \mathrm{IV})$ y ranitidina $(1 \mathrm{mg} / \mathrm{kg}$, SC), excepto en el protocolo 1, donde primero se aplicaron los fármacos de premedicación y 20 min después los antibióticos y la ranitidina. Finalmente se procedió a la limpieza preoperatoria de la zona quirúrgica del paciente.

- La segunda medición se realizó en el periodo de inducción, aplicándose los fármacos respectivos de cada protocolo anestésico que inducen al paciente en el plano anestésico quirúrgico necesario. La medición se realizó 2 min después de aplicado los agentes anestésicos de inducción.

- La tercera medición se realizó en el periodo de mantenimiento del plano anestésico mediante infusión continua (TIVA). En el protocolo 4, se adicionó el anestésico inhalatorio con oxígeno. Las mediciones de las funciones fisiológicas se hicieron cada $5 \mathrm{~min}$, obteniéndose un promedio para cada paciente.
- La cuarta medición se realizó al término de la cirugía. La medición se efectuó a los 5 min de concluida la cirugía y de retirado el mantenimiento anestésico.

La medición de los valores para la variable saturación de oxígeno se realizó únicamente en el periodo de mantenimiento y al término de la cirugía.

\section{Evaluación Estadística}

Se realizó el análisis de varianza para las constantes vitales y se hizo la prueba de Tuckey con una confiabilidad del 95\%, para comparar los resultados obtenidos entre los distintos protocolos. Las variables analizadas y los valores esperados fueron: frecuencia cardiaca de 61 a 160 latidos por minuto (Belerenian et al., 2001; Engel y García, 2008; Rioja et al., 2013), frecuencia respiratoria de 11 a 30 respiraciones por minuto (Engel y García, 2008), saturación de oxígeno de $(<75$, $76-90,>90 \%$ intervalo de $\mathrm{SO}_{2}$ (Thurmon et al., 2003) y temperatura corporal de 38.0 a $39 .{ }^{\circ} \mathrm{C}$ (Báez et al., 2007; Engel y García, 2008).

\section{Resultados y Discusión}

No se llegó a obtener un plano anestésico quirúrgico en el protocolo 2 (se esperó durante cinco minutos) en ninguno de los ocho pacientes, por lo que se agregó un bolo de carga de xilacina a dosis de $0.9 \mathrm{mg} / \mathrm{kg}$ para obtener dicho plano. Asimismo, en el protocolo 4 se tuvo que usar una concentración de isoflurano al $1.5 \%$ para lograr un buen mantenimiento anestésico.

\section{Frecuencia Cardiaca}

En el periodo de inducción se encontró diferencia significativa $(\mathrm{p}<0.05)$ entre el protocolo 4 con los protocolos 1 y 2 (Cuadro 1 ). En el protocolo 2 se presentaron tres pacientes con taquicardia, en tanto que cinco pacientes del protocolo 1 presentaron taqui- 
Cuadro 1. Frecuencia cardiaca (FC/min) (promedio \pm desviación estándar) en pacientes caninos $(n=8)$ durante los cuatro periodos anestésicos, según el protocolo de anestesia

\begin{tabular}{ccccc}
\hline Protocolo $^{1}$ & Preoperatorio & Inducción & Mantenimiento & Término \\
\hline 1 & $132.4 \pm 37.4$ & $163.6 \pm 27.8^{\mathrm{a}}$ & $90.0 \pm 35.5^{\mathrm{ab}}$ & $80.6 \pm 32.7$ \\
2 & $113.3 \pm 38.8$ & $147.4 \pm 32.9^{\mathrm{a}}$ & $93.0 \pm 39.8^{\mathrm{ab}}$ & $88.4 \pm 31.7$ \\
3 & $127.0 \pm 34.6$ & $131.6 \pm 31.6^{\mathrm{ab}}$ & $107.0 \pm 32.6^{\mathrm{b}}$ & $90.6 \pm 5.1$ \\
4 & $105.8 \pm 37.2$ & $90.4 \pm 25.2^{\mathrm{b}}$ & $71.0 \pm 18.5^{\mathrm{a}}$ & $80.6 \pm 32.7$ \\
\hline
\end{tabular}

a,b Superíndices diferentes dentro de columnas indican diferencia estadística $(p<0.05)$

1 Protocolo 1: acepromacina, morfina, diazepam, ketamina y xilacina; Protocolo 2: diazepam, ketamina, xilacina; Protocolo 3: propofol, diazepam, fentanilo; Protocolo 4: propofol, isoflurano, fentanilo

cardia. La hipotensión generada por la acepromacina pudo provocar taquicardia refleja (Gonzalo et al., 1994), así como la ketamina (Engel y García, 2008; Plumb, 2010). Los pacientes del protocolo 4 obtuvieron un pequeño descenso en su FC, pero dentro de los niveles fisiológicos normales (Cuadro 1), posiblemente debido al propofol (Plumb, 2010; Rioja et al., 2013).

En el periodo de mantenimiento se halló diferencia significativa entre los protocolos 3 y 4, donde el mayor registro de FC registrado para el protocolo 3 se atribuye como acción refleja a la depresión sobre la $\mathrm{SpO}_{2}$ causada por el propofol (Rioja et al., 2013). No obstante, la FC en ambos protocolos se mantuvo dentro de los parámetros normales (Cuadro 1). Hallazgos similares han sido reportados por Peña et al. (2007) para la inducción con propofol.

Un paciente, tanto del protocolo 1 como del 4, presentó bradicardia, lo cual es atribuido a la acción depresora de la xilacina sobre el aparato cardiovascular (Laredo et al., 2001b; Muir et al., 2008; Rioja et al., 2013) y la depresión miocárdica causada por el isoflurano (Plumb, 2010), respectivamente. En forma similar, al término de la cirugía, pacien- tes de los protocolos 1, 2 y 4 (uno por protocolo) presentaron bradicardia.

\section{Pulso}

Se encontró diferencia significativa para el periodo de mantenimiento entre el protocolo 3 y 4 , donde los pacientes del protocolo 3 presentaron un mayor registro de pulsaciones por minuto respecto al protocolo 4 , pero siempre dentro de los valores normales. En forma similar a la FC, la depresión causada sobre la $\mathrm{SpO}_{2}$ por el propofol incrementaría la velocidad de las pulsaciones (Cuadro 2). Asimismo, durante el periodo de mantenimiento, dos pacientes, tanto del protocolo 2 como del protocolo 4, presentaron pulsos hipocinéticos (valores entre 49 y 59 pulsaciones por minuto).

En el Cuadro 2 se observa que los valores promedio de pulso en los pacientes del protocolo 4 se comportan de una manera muy similar en el preoperatorio y durante la inducción, pero caen significativamente durante la fase de mantenimiento $(\mathrm{p}<0.05)$ para recuperarse al término de la cirugía. Esto es una indicación que la dosis anestésica aplicada estaría siendo metabolizada, especialmente del isoflurano (Laredo et al., 2001a; Palacios y Benítez, 2005). 
Cuadro 2. Pulsaciones por minuto (promedio \pm desviación estándar) en pacientes caninos $(n=8)$ durante los cuatro periodos anestésicos, según el protocolo de anestesia

\begin{tabular}{ccccc}
\hline Protocolo $^{1}$ & Preoperatorio & Inducción & Mantenimiento & Término \\
\hline 1 & $130.3 \pm 30.1$ & $123.0 \pm 33.3$ & $90.6 \pm 31.6^{\mathrm{ab}}$ & $83.9 \pm 24.7$ \\
2 & $112.9 \pm 36.2$ & $122.5 \pm 32.6$ & $91.8 \pm 42.0^{\mathrm{ab}}$ & $86.5 \pm 23.7$ \\
3 & $109.4 \pm 47.1$ & $115.4 \pm 35.3$ & $106.1 \pm 33.3^{\mathrm{a}}$ & $90.6 \pm 5.1$ \\
4 & $99.8 \pm 29.6$ & $92.0 \pm 31.3$ & $68.6 \pm 22^{\mathrm{b}}$ & $83.0 \pm 35.3$ \\
\hline
\end{tabular}

a,b Superíndices diferentes dentro de columnas indican diferencia estadística $(p<0.05)$

1 Protocolo 1: acepromacina, morfina, diazepam, ketamina y xilacina; Protocolo 2: diazepam, ketamina, xilacina; Protocolo 3: propofol, diazepam, fentanilo; Protocolo 4: propofol, isoflurano, fentanilo

Cuadro 3. Frecuencia respiratoria $(\mathrm{FR} / \mathrm{min})$ (promedio \pm desviación estándar) en pacientes caninos $(n=8)$ durante los cuatro periodos anestésicos, según el protocolo de anestesia

\begin{tabular}{ccccc}
\hline Protocolo $^{1}$ & Preoperatorio & Inducción & Mantenimiento & Término \\
\hline 1 & $50.5 \pm 29.3$ & $24.4 \pm 19.5$ & $28.6 \pm 31.1$ & $30.3 \pm 38.6$ \\
2 & $35.3 \pm 28.9$ & $27.0 \pm 26.4$ & $33.8 \pm 32.9$ & $38.6 \pm 33.4$ \\
3 & $51.8 \pm 27.4$ & $39.3 \pm 28.2$ & $30.1 \pm 38.8$ & $18.4 \pm 21.4$ \\
4 & $44.4 \pm 33.0$ & $34.9 \pm 29.0$ & $30.1 \pm 31.7$ & $34.9 \pm 29.0$ \\
\hline
\end{tabular}

1 Protocolo 1: acepromacina, morfina, diazepam, ketamina y xilacina; Protocolo 2: diazepam, ketamina, xilacina; Protocolo 3: propofol, diazepam, fentanilo; Protocolo 4: propofol, isoflurano, fentanilo

\section{Frecuencia Respiratoria}

No se encontraron diferencias significativas en frecuencia respiratoria entre los protocolos (Cuadro 3); sin embargo, durante el periodo de mantenimiento, un paciente del protocolo 4 presentó bradipnea debido al efecto depresor del isoflurano sobre la frecuencia respiratoria (Laredo et al., 2001a; Palacios y Benítez, 2005; Sumano y Ocampo, 2006). Asimismo, un paciente del protocolo 2 y uno del protocolo 4 presentaron taquipnea intensa en la fase de mantenimiento y, en forma similar, un paciente del protocolo 2 y dos del protocolo 4 presentaron taquipnea inten- sa al término de la cirugía, lo cual es atribuible a la falta de analgesia (Engel y García, 2008).

El comportamiento de la frecuencia respiratoria en los protocolos 2 y 4 (Cuadro 3) fue similar, manteniéndose prácticamente por encima de los límites normales durante todos los periodos de la cirugía, indicando una deficiente analgesia (Engel y García, 2008). El protocolo 3 presentó un descenso continuo y depresión respiratoria pasajera (Muir et al., 2008) ocasionada por el propofol (Plumb, 2010), pero dentro de los límites normales. Similares resultados se obtuvieron para el 
propofol por Thibaut et al. (2002) y Peña et al. (2007).

\section{Saturación de Oxígeno}

Se encontró diferencia significativa $(\mathrm{p}<0.05)$ para el periodo de mantenimiento entre protocolos, excepto entre el protocolo 1 y 2. El protocolo 3 presentó los índices más bajos de $\mathrm{SpO}_{2}$ (Cuadro 4), ya que cuatro pacientes en el protocolo 3 presentaron hipoxemia muy grave y otros cuatro presentaron hipoxemia grave, siendo necesario realizar ventilación asistida hasta mejorar el índice de $\mathrm{SpO}_{2}$. Esta deficiencia en la saturación de oxígeno se atribuye al propofol y fentanilo (Laredo y Cantalapiedra, 2001; Rioja et al., 2013). Resultados similares fueron encontrados por Peña et al. (2007).

Los pacientes del protocolo 1 y 2 estuvieron próximos al límite inferior de una $\mathrm{SpO}_{2}$ normal. Mejores registros se obtuvieron en pacientes del protocolo 1, posiblemente debido a que en el protocolo 2 se aplicó xilacina como agente inductor, provocando menor $\mathrm{SpO}_{2}$ (Thurmon et al., 2003).

También se encontró diferencia significativa para el periodo de término de la cirugía entre protocolos, excepto entre el protocolo 1 y 2 . El protocolo 3 mostró una recuperación rápida en cuanto a su deficiente índice de SpO2 (Cuadro 4) hallándose siete pacientes con hipoxemia grave y uno con $\mathrm{SpO} 2$ normal. Esto se debe a la rápida velocidad de metabolización que posee el propofol (Plumb, 2007; Rioja et al., 2013).

El protocolo 1 y 2 también mostraron una recuperación en su $\mathrm{SpO}$, mostrando mejores valores para el protocolo 1 .

El protocolo 4 fue el que mostró un mejor índice de saturación de oxígeno, debido probablemente a la técnica de anestesia inhalatoria (Laredo et al., 2001a; Palacios y Benítez, 2005; Rioja et al., 2013).

\section{Temperatura Corporal}

Se observó un descenso gradual de la temperatura en todos los casos (Cuadro 5). Se halló diferencia significativa para los periodos de inducción y mantenimiento entre los protocolos 1 y 2 , habiendo un mejor control de la temperatura corporal en los pacientes del protocolo 2. En el periodo de mantenimiento, uno de los pacientes del protocolo 1 registró una temperatura muy baja $\left(36^{\circ} \mathrm{C}\right)$, atribuida a la acepromacina (Plumb, 2010). Asimismo, un paciente del protocolo $1 \mathrm{y}$ uno del protocolo 4 presentaron temperaturas bajas $\left(35.4\right.$ y $36^{\circ} \mathrm{C}$, respectivamente) al término de la cirugía, lo cual estuvo generado por la acepromacina y morfina en el primer caso e isoflurano en el segundo caso (Engel y García, 2008; Plumb, 2010).

Resultados similares fueron encontrados para esta variable por Peña et al. (2007). Otros trabajos sobre protocolos anestésicos como los realizados por Otero et al. (2000), Báez et al. (2007); Da Costa et al. (2011) y Albuquerque et al. (2015) también encontra-

Cuadro 4. Saturación de oxígeno (\%) (promedio \pm d.e.) en pacientes caninos $(n=8)$ durante el mantenimiento del plano anestésico y al término de la cirugía, según el protocolo de anestesia

\begin{tabular}{|c|c|c|}
\hline Protocolo $^{1}$ & Mantenimiento & Término \\
\hline 1 & $90.0 \pm 6.5^{\mathrm{a}}$ & $91.6 \pm 6.1^{\mathrm{a}}$ \\
\hline 2 & $86.0 \pm 7.7^{\mathrm{a}}$ & $90.8 \pm 6.1^{\mathrm{a}}$ \\
\hline 3 & $75.0 \pm 7.8^{b}$ & $85.9 \pm 4.3^{b}$ \\
\hline 4 & $97.0 \pm 9.8^{c}$ & $97.0 \pm 1.5^{\mathrm{c}}$ \\
\hline \multicolumn{3}{|c|}{ a,b,c Superíndices diferentes dentro de columnas } \\
\hline \multicolumn{3}{|c|}{$\begin{array}{l}1 \text { Protocolo 1: acepromacina, morfina, dia- } \\
\text { zepam, ketamina y xilacina; Protocolo 2: dia- } \\
\text { zepam, ketamina, xilacina; Protocolo 3: pro- } \\
\text { pofol, diazepam, fentanilo; Protocolo 4: pro- } \\
\text { pofol, isoflurano, fentanilo }\end{array}$} \\
\hline
\end{tabular}


Cuadro 5. Temperatura corporal $\left({ }^{\circ} \mathrm{C}\right)$ (promedio \pm desviación estándar) en pacientes caninos $(n=8)$ durante los cuatro periodos anestésicos, según el protocolo de anestesia

\begin{tabular}{ccccc}
\hline Protocolo $^{1}$ & Preoperatorio & Inducción & Mantenimiento & Término \\
\hline 1 & $38.8 \pm 0.3$ & $37.7 \pm 1.1^{\mathrm{a}}$ & $37.0 \pm 1.0^{\mathrm{a}}$ & $37.0 \pm 1.1$ \\
2 & $38.8 \pm 0.7$ & $38.6 \pm 0.1^{\mathrm{b}}$ & $38.0 \pm 0.8^{\mathrm{b}}$ & $37.8 \pm 1.1$ \\
3 & $38.9 \pm 0.7$ & $38.2 \pm 0.3^{\mathrm{ab}}$ & $38.0 \pm 0.8^{\mathrm{ab}}$ & $37.6 \pm 1.2$ \\
4 & $38.8 \pm 0.2$ & $38.2 \pm 0.8^{\mathrm{ab}}$ & $38.0 \pm 0.8^{\mathrm{ab}}$ & $37.8 \pm 0.9$ \\
\hline
\end{tabular}

a,b Superíndices diferentes dentro de columnas indican diferencia estadística $(p<0.05)$

${ }^{1}$ Protocolo 1: acepromacina, diazepam, ketamina y xilacina; Protocolo 2: diazepam, ketamina, xilacina; Protocolo 3: propofol, diazepam, fentanilo; Protocolo 4: propofol, isoflurano, fentanilo

ron disminución de la temperatura en forma constante a lo largo de la anestesia.

Es común que los pacientes pierdan entre 1 y $4{ }^{\circ} \mathrm{C}$ durante la anestesia. Los fármacos anestésicos producen vasodilatación aumentando la posibilidad de pérdida de calor, como también el hecho de rasurar el pelo de los pacientes y preparar la piel con soluciones no atemperadas (Rioja et al., 2013).

\section{Conclusiones}

- Los mejores planos anestésicos se obtuvieron con los protocolos 1 y 3 .

- El protocolo 2 registró diversas alteraciones de la frecuencia cardiaca.

- Los protocolos 2 y 4 no presentaron un nivel adecuado de analgesia.

- El protocolo 3 afectó la saturación de oxígeno.

- El protocolo 1 presentó el mayor efecto depresor sobre la temperatura corporal.

\section{Agradecimientos}

El autor expresa su agradecimiento al Mag. Guillermo Vásquez Rodríguezy al M.V.Z. Alexander Ureta Escobedo por su asesoría en la realización de este trabajo de investigación. Asimismo, a la Clínica Veterinaria Terán por las facilidades brindadas en la realización de este trabajo. Se agradece especialmente a los egresados de Medicina Veterinaria Catalina Guzmán Ríos, Gianfranco Díaz Vargas y Anggy Gutiérrez Trillo, a la Srta. Kathy Paquico, y a la Arq. Patricia Rivas Vargas.

\section{Literatura Citada}

1. [ASA] American Society of Anesthesiologists. 2014. Standards and Guidelines. [Internet]. Available in: http:/ /www.asahq.org/quality-and-practicemanagement/standards-and-guidelines

2. Albuquerque V, Araújo M, Ferreira $G$, Fonseca M, Abimussi C, Ferreira J, Oliva V.2015. Epidural levobupivacaine alone or combined with different morphine doses in bitches under continuous propofol infusion. Arq Bras Med Vet Zootec 67: 951-960. doi: 10.1590/1678-4162-7661

3. Báez P, Ruiz I, Restrepo L, Ruiz J. 2007. Comparación de dos protocolos anestésicos para ovariohisterectomía en perras sanas. Rev Colomb Cienc Pec 20: 425-430.

4. Belerenian G, Mucha C, Camacho A. 2001. Afecciones cardiovasculares en pequeños animales. Buenos Aires, Argentina: Inter-Médica. $360 \mathrm{p}$.

5. Burzaco O, Martínez M. 2001. La valoración preanestésica. Riesgo anestésico. Consulta Difus Vet 9(78): 49-62. 
6. Cantalapiedra A, Cruz J. 2001. Monitorización anestésica en los pequeños animales. Consulta Difus Vet 9(77): 97-104.

7. Da Costa J, Ramirez R, Lima R, Isidro P, Alves M. 2011. Control de hipotermia con colchón térmico en perras durante ovario-histerectomía. Rev Med Vet 22: 11-19. doi: 10.19052/mv.559

8. Da Silva J, Viera R, Xavier R, Pereira A. 2000. Anestesia por infusão contínua de propofol em cães pré-medicados com acepromazina e fentanil. Cienc Rural 30: 829-834.

9. Engel J, García L. 2008. Manual del ATV (auxiliar técnico veterinario). Barcelona, España: Multimédica. $447 \mathrm{p}$.

10. Fossum T. 2009. Cirugía en pequeños animales. $3^{\mathrm{a}}$ ed. España: Elsevier. 1610 p.

11. Gonzalo J, Avila I, Bonafonte I, García F, Orden A, Pereira J, et al. 1994. Cirugía veterinaria. Madrid, España: McGraw-Hill. 884 p. 